\title{
Dramatic expansion of the black widow toxin arsenal uncovered by multi-tissue transcriptomics and venom proteomics
}

\author{
Robert A Haney ${ }^{1}$, Nadia A Ayoub², Thomas H Clarke², Cheryl Y Hayashi ${ }^{3}$ and Jessica E Garb ${ }^{*}$
}

\begin{abstract}
Background: Animal venoms attract enormous interest given their potential for pharmacological discovery and understanding the evolution of natural chemistries. Next-generation transcriptomics and proteomics provide unparalleled, but underexploited, capabilities for venom characterization. We combined multi-tissue RNA-Seq with mass spectrometry and bioinformatic analyses to determine venom gland specific transcripts and venom proteins from the Western black widow spider (Latrodectus hesperus) and investigated their evolution.

Results: We estimated expression of 97,217 L. hesperus transcripts in venom glands relative to silk and cephalothorax tissues. We identified 695 venom gland specific transcripts (VSTs), many of which BLAST and GO term analyses indicate may function as toxins or their delivery agents. 38\% of VSTs had BLAST hits, including latrotoxins, inhibitor cystine knot toxins, CRISPs, hyaluronidases, chitinase, and proteases, and 59\% of VSTs had predicted protein domains. Latrotoxins are venom toxins that cause massive neurotransmitter release from vertebrate or invertebrate neurons. We discovered $\geq 20$ divergent latrotoxin paralogs expressed in $L$. hesperus venom glands, significantly increasing this biomedically important family. Mass spectrometry of $L$. hesperus venom identified 49 proteins from VSTs, 24 of which BLAST to toxins. Phylogenetic analyses showed venom gland specific gene family expansions and shifts in tissue expression.

Conclusions: Quantitative expression analyses comparing multiple tissues are necessary to identify venom gland specific transcripts. We present a black widow venom specific exome that uncovers a trove of diverse toxins and associated proteins, suggesting a dynamic evolutionary history. This justifies a reevaluation of the functional activities of black widow venom in light of its emerging complexity.
\end{abstract}

Keywords: RNA-Seq, Latrotoxins, Venom, Mass spectrometry, Transcriptomics, Spider

\section{Background}

Venomous taxa have evolved many times within the metazoa [1], and occur in both vertebrates and invertebrates. The venoms these diverse taxa produce are chemically complex and play key roles in organismal ecology, functioning in both predation and defense. Molecules contributing to the toxicity of venom are the focus of sustained effort aimed at characterizing their physiological roles and biochemical action, given their potential in pharmacological and biomedical applications [2]. Venom toxins are often members of large gene families, and the study of

\footnotetext{
* Correspondence: Jessica_Garb@uml.edu

'Department of Biological Sciences, University of Massachusetts, Lowell, MA 01854, USA

Full list of author information is available at the end of the article
}

their evolution can illuminate the roles of gene duplication, convergence and positive selection in generating the functional diversity of venoms [3]. Determining the molecular diversity of venoms is the necessary first step in this process, yet few studies have utilized large scale approaches for venom characterization.

Spiders (Order Araneae) are the most species-rich venomous clade, with $>44,000$ described species [4], the overwhelming majority of which are venomous. Estimates of the number of unique venom peptides and proteins produced by members of this clade range from 1.5 - 20 million [5-7], significantly more than are estimated from other major clades of venomous invertebrates such as scorpions and cone snails $[8,9]$. The venoms of some spiders have been extensively studied, largely due to the 
potential for isolating novel insecticidal toxins [7], and reasons of direct medical concern [10-13]. However, past work has focused on a small fraction of total spider species, and much of the molecular diversity of spider venoms remains to be discovered.

Spider venom proteins characterized to date belong to several different broad classes: enzymes (such as proteases, phospholipases and hyaluronidases), small linear cytolytic peptides, and neurotoxins with differing functionality and size range [7]. The most commonly documented form of spider neurotoxin is a small $(<15 \mathrm{kDa})$, disulfide-rich peptide. The disulfide bonds give rise to one of three typical structural motifs, the disulfide-directed $\beta$-hairpin, the Kunitz motif, or the inhibitor cystine knot (ICK), the last of which appears to be the most common amongst studied spider venoms [14]. The compact structure of ICK peptides renders them highly resistant to the actions of proteases in envenomated organisms, contributing to their efficacy [15]. Different ICK peptides specifically target different ion channels in the nervous system [11], and diverse sets of these peptides can occur within the venom of even a single species [14,12], acting synergistically with one another and with small linear peptides $[14,16,17]$ in a manner similar to the "toxin cabals" of cone snails [18].

The most prominent exception to this venom smallmolecule $(<15 \mathrm{kDa})$ dominance occurs in the black widow spiders (genus Latrodectus, family Theridiidae), which contain multiple large ( $>130 \mathrm{kDa})$ neurotoxic proteins known as latrotoxins, encoded by paralogous loci [19-26]. The best studied of the latrotoxins, $\alpha$-latrotoxin, forms tetrameric complexes which bind to vertebrate presynaptic receptors and insert into neuronal membranes, forming calcium-permeable ion channels that stimulate massive neurotransmitter release [27]. $\alpha$-Latrotoxin is also widely known as the causative agent of the extreme pain associated with black widow bites. Other functionally characterized latrotoxins differ in their phyletic specificity, affecting the nervous systems of only insects or crustaceans. Latrotoxin proteins are accompanied in the venom by lowmolecular weight peptides called latrodectins (also known as $\alpha$-latrotoxin associated LMWPs) that may enhance latrotoxin toxicity $[20,28]$, although they exhibit no toxicity themselves [29].

Given the large number of peptides and proteins remaining to be discovered in the venoms of spider species, next generation RNA sequencing (RNA-Seq) methods are particularly well suited for rapidly obtaining a comprehensive inventory of venom components, as well as an improved functional understanding of the venom gland. The high-throughput of next-generation sequencing allows for profiling of transcripts over a wide range of abundance [30], providing an accurate picture of differential expression across tissues within an organism. A multi-tissue approach allows for the identification of transcripts with highly biased expression in the venom gland, whose products are candidates for function in the venom as toxins, or in venom production. Venom gland specific sequences can then be subjected to bioinformatic and evolutionary analyses to discover novel toxins and to better understand their origins and the mechanisms generating their diversity. The insights provided by transcriptomic data can be greatly enhanced by proteomics approaches which permit a direct examination of the peptide and protein composition of venoms, typically with methods coupling liquid chromatography based separation to mass spectrometry [6]. These methods have begun to be applied to a range of species, leading to an expansion of the number of venom peptide and protein toxins known from arachnids $[31,32]$.

In this study we present an integrated set of multi-tissue transcriptomic and proteomic data from the Western black widow spider, Latrodectus hesperus, to investigate the composition and evolution of its venom. The venom of this species remains largely unexplored, despite the relevance of black widows to human health and the importance of their venom in studies of vertebrate neurotransmission [33-35]. We identify transcripts with biased expression in the venom gland relative to other tissues, and potential toxin transcripts in the venom gland exome, using bioinformatics-based approaches. We also explore the relative abundance of transcripts specific to the venom gland and quantify the representation of the biological functions and processes in which these transcripts take part. We identify prominent toxin families, and perform phylogenetic analyses to investigate their evolution. Lastly, we explicitly identify the secreted peptide and protein component of the venom using a mass spectrometric based proteomic approach. Our transcriptome and proteome provide complementary data in order to separate the secreted venom components from the cast of molecules that support toxin production within the gland.

\section{Results}

\section{Bioinformatic functional categorization of the $L$. hesperus venom gland transcriptome}

RNA-Seq libraries were constructed and sequenced from three L. hesperus tissue types: (1) venom glands (52,044,562 reads), (2) silk glands (15,093,424 reads), (3) cephalothorax with venom glands removed (50,969,807 reads). Sequencing reads from each tissue-specific library were separately assembled with Trinity and the three transcript sets were merged with CAP3 [36] to produce a non-redundant set of sequences. This resulted in a transcriptome comprised of 103,635 sequences, 97,217 of which were retained as Unique Assembled Transcripts (UATs) after filtering out sequences encoding identical proteins (see also [37]). Transcripts were submitted to a suite of analyses to investigate their identity, diversity and function in the venom gland, as well as the presence of their products in the 
venom (Figure 1). Six-hundred ninety-five $(0.71 \%)$ of the 97,217 transcripts had expected venom gland counts per million (eCPM) greater than one and were either exclusively expressed in the venom gland (386 transcripts), or were among the top $2.5 \%$ in the distribution of the ratio of venom gland eCPM values to both silk and cephalothorax. This equates to a level of expression in the venom gland that is at least 306-fold higher than that in silk, and at least 32-fold higher than in cephalothorax. We hereafter refer to this set of 695 sequences as venom gland specific transcripts (VSTs). Of the VSTs, 266 (38.3\%) had a significant (e-value $\leq 1 \mathrm{e}-5)$ BLASTx hit to the UniProt database, while 429 (61.7\%) had no significant BLASTx hit to UniProt at this e-value cutoff (Additional file 1). Among VSTs were 45 with significant BLAST similarity to known venom toxins, as well as to 17 enzymes that may act to facilitate toxin action (Table 1, Additional file 1). A total of 1312 GO terms were mapped to 228 sequences in the VST set. GOseq analysis recovered 18 GO terms that were overrepresented in the VSTs compared to all transcripts with an
$\mathrm{eCPM}>1$ at a false discovery rate (FDR) cutoff of 0.05 (Table 2). Overrepresented categories for VSTs in the cellular component ontology included (1) extracellular region, (2) other organism cell membrane and (3) other organism presynaptic membrane. Exocytosis and proteolysis were significantly overrepresented categories in the biological process ontology, while serine endopeptidase and metalloprotease activity were among the overrepresented categories in the molecular function ontology.

Of the 695 VSTs, 414 had at least one protein domain prediction from InterProScan, including 179 sequences with no significant BLAST hit at UniProt. Among all protein domains identified more than five times amongst the VSTs, ankyrin domains were most common, while leucine-rich repeat, low density lipoprotein receptor class $\mathrm{A}$, immunoglobulin, chitin-binding, helix loop helix, latrotoxin C-terminal, venom allergen 5, serine protease and metalloprotease domains also commonly occurred in predicted proteins from the VST set (Additional file 2, Additional file 3).

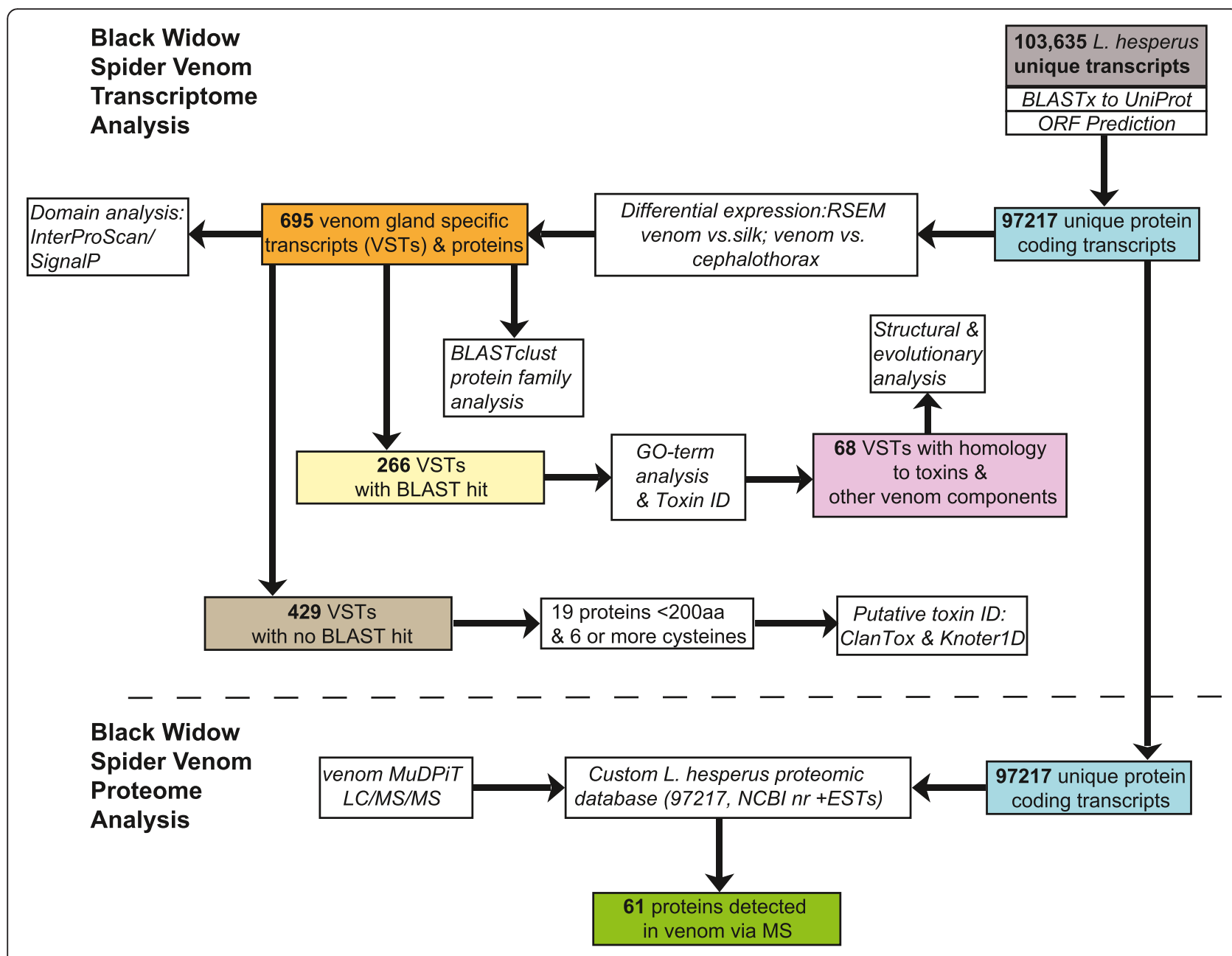

Figure 1 Flowchart of analyses performed on the set of $L$. hesperus venom gland specific transcripts (VSTs). Colored boxes indicate subsets of sequences resulting from specific analyses. Boxes below the dashed line indicate analyses with the combined proteomic and transcriptomic datasets. 
Table 1 Summary of groups of toxins and enzymes in L. hesperus venom gland specific transcripts

\begin{tabular}{lllll}
\hline Type & Number $^{\mathbf{a}}$ & \% expression & Proteomic evidence & Proposed function \\
\hline Latrodectins & 2 & 16.1 & Yes & Facilitate latrotoxin action \\
Latrotoxins & $39(20)$ & 15.8 & Yes & Form calcium channels, cause neurotransmitter release in target organism \\
Putative ICK & 7 & 14.2 & Yes & Potential ion channel action \\
CRISPs & $2 / 3$ & 8.4 & Yes & Calcium channel blocker, smooth muscle paralysis \\
Metalloproteases & $8 / 11$ & 7.8 & Yes & Tissue degradation/spreading factor \\
ICK toxins & 4 & 5.4 & Yes & Alter function of neuronal ion channels \\
Serine proteases & $6 / 7$ & 2.8 & Yes & Tissue degradation/ spreading factor \\
Chitinases & 1 & 1.6 & Yes & Breakdown of arthropod exoskeleton \\
LRR proteins & $8 / 9$ & 0.82 & Yes & Diverse/neural development \\
Hyaluronidases & 2 & 0.5 & Yes & Breakdown of extracellular matrix/spreading factor \\
\hline
\end{tabular}

${ }^{a}$ The number preceding the slash is the total number of sequences with a BLAST hit to the defined type, while the number after the slash indicates the total number of sequences in this group including additional sequences identified in clustering analysis, but without a top BLAST hit to this sequence type. The number in parentheses indicates the minimum number of putative distinct latrotoxin paralogs. The number of putative ICKs is shown in italic as it is not based on BLAST homology (see text). For each type, the total number of sequences, \% of total expression among all venom gland specific transcripts for all sequences of this type, the proteomic evidence for the presence of the type in venom and the proposed function are shown.

\section{L. hesperus toxin diversity and evolution Latrotoxins}

The majority of the diversity among VSTs with BLAST homology to known toxins was contributed by latrotoxins. Strikingly, the number of distinct sequences found suggests a wider range of latrotoxin diversity than previously reported $[38,13]$. A total of 39 VST sequences were identified as latrotoxins. Latrotoxins are large proteins, and range from $\sim 1200$ to 1400 amino acids in length
[19-22], posing a challenge for transcript reconstruction from short-read data. The predicted latrotoxin proteins from the $L$. hesperus transcriptome varied in length, and many appear to be fragments of larger proteins. We aligned the 20 latrotoxin sequences in the set with a predicted protein of at least 500 amino acids with ten additional complete or near full-length latrotoxin sequences from Latrodectus and Steatoda species from the NCBI $n r$ database. A Bayesian phylogenetic tree produced from this

Table 2 Overrepresented GO terms in the $L$. hesperus venom gland specific transcript set

\begin{tabular}{|c|c|c|c|}
\hline GOID & Ontology & Term & p-value \\
\hline GO:0044218 & CC & other organism cell membrane & $1.17 \mathrm{e}-73$ \\
\hline GO:0044279 & CC & other organism membrane & $1.17 e-73$ \\
\hline GO:0072556 & CC & other organism presynaptic membrane & $1.17 e-73$ \\
\hline GO:0005576 & $\mathrm{CC}$ & extracellular region & $7.27 e-40$ \\
\hline GO:0016021 & CC & integral to membrane & $4.70 e-12$ \\
\hline GO:0016020 & CC & membrane & $2.61 \mathrm{e}-10$ \\
\hline GO:0006887 & $\mathrm{BP}$ & exocytosis & $2.42 \mathrm{e}-68$ \\
\hline GO:0019079 & $\mathrm{BP}$ & viral genome replication & $2.26 \mathrm{e}-07$ \\
\hline GO:0006508 & $\mathrm{BP}$ & proteolysis & $2.96 \mathrm{e}-07$ \\
\hline GO:0080009 & $\mathrm{BP}$ & mRNA methylation & $2.65 e-05$ \\
\hline GO:0042302 & MF & structural constituent of cuticle & $3.51 \mathrm{e}-12$ \\
\hline GO:0008237 & MF & metallopeptidase activity & $9.73 e-08$ \\
\hline GO:0004222 & MF & metalloendopeptidase activity & $1.56 \mathrm{e}-07$ \\
\hline GO:0003968 & MF & RNA-directed RNA polymerase activity & $4.22 \mathrm{e}-06$ \\
\hline GO:0004252 & MF & serine-type endopeptidase activity & $4.78 \mathrm{e}-06$ \\
\hline GO:0008174 & MF & mRNA methyltransferase activity & $9.57 \mathrm{e}-06$ \\
\hline GO:0004089 & MF & carbonate dehydratase activity & $1.62 \mathrm{e}-05$ \\
\hline GO:0004415 & MF & hyalurononglucosaminidase activity & $1.08 \mathrm{e}-04$ \\
\hline
\end{tabular}

All significant terms at an FDR $\leq 0.05$ are shown with brief term descriptions, separated into groups by ontology. $\mathrm{CC}=$ cellular component, $\mathrm{BP}=$ biological process, MF = molecular function. 
alignment included four sub-clades each containing one of the four functionally characterized latrotoxins from L. tredecimguttatus, together with conserved orthologs assembled from our L. hesperus short-read data (Figure 2). However, the majority of $L$. hesperus sequences we assembled with homology to latrotoxins were not contained within these four clades and were instead dispersed among multiple highly supported clades. The maximum-likelihood topology was identical to the Bayesian tree with the exception of the placement of two sequences (Contig 2336 and venom_comp_1099970_c1_seq1) as unresolved branches at the base of the tree, instead of together in a clade (Figure 2).

Seven latrotoxin protein sequences inferred from $L$. hesperus VSTs possessed a start codon as well as the distinctive latrotoxin C-terminal domain [38], followed by a 3' UTR, suggesting that they are full-length or near full-length copies. We examined the domain structure of these putative full-length latrotoxins. Multiple ankyrin repeats (protein-protein interaction motifs with a helix-loop-helix structure [39]) were predicted in each of these sequences, and the number of repeats ranged from 11 to 20 per sequence. Variation was also evident when comparing $L$. hesperus protein sequences with the functionally characterized orthologs from $L$. tredecimguttatus (Figure 3). One sequence, venom_comp106397_c0_seq1 (labeled 1 in Figure 3), groups closely with L. tredecimguttatus $\delta$-latroinsectotoxin (sequence 2), but has 14 repeats, as opposed to 13 in the published sequence from L. tredecimguttatus [21]. The L. hesperus ortholog of $\alpha$-latroinsectotoxin has 20 ankyrin repeats, as does that of L. tredecimguttatus. Other novel L. hesperus latrotoxin sequences had either 11 or 17 ankyrin repeats (Figure 3 ).

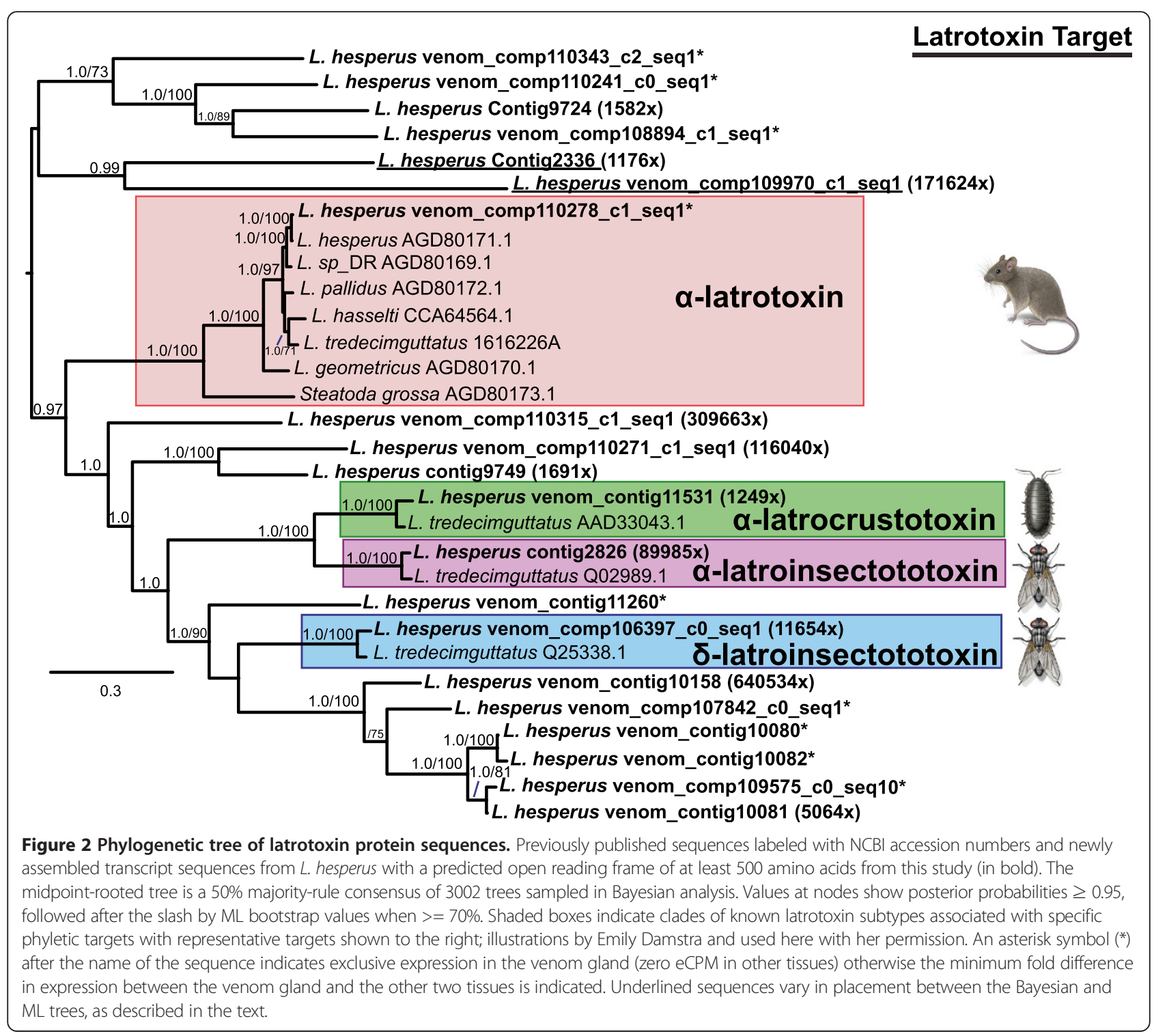




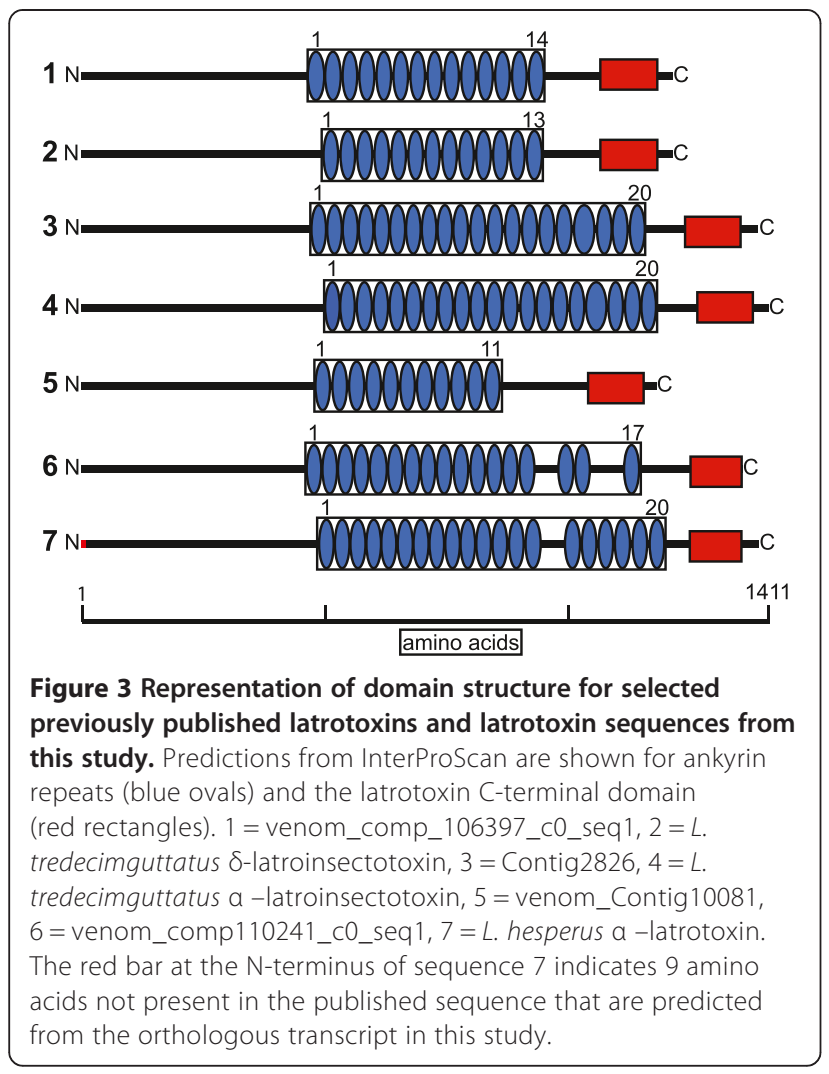

We searched the entire translated $L$. hesperus transcriptome to identify other sequences with homology to latrotoxins, but lacking venom gland biased expression. As ankyrin domains are common components of many nonhomologous proteins with diverse functions, we limited the BLASTp search to the conserved and distinct Nterminus of the latrotoxin protein, which lacks ankyrin repeats. Two hits were recovered. However, read count data indicate that they lack expression in tissues other than venom gland, and were not included as VSTs because they did not reach the minimum read count threshold for inclusion. These two sequences were not included in phylogenetic analyses, as they did not meet the minimum length requirement.

\section{ICK toxins and other small proteins with potential toxicity}

The most common type of toxin in many spider venoms, as well as in scorpion, cone snail and remipede venoms [40] are small disulfide-bonded neurotoxins with an inhibitor cystine knot (ICK) structural motif. Previously, ICK toxins were not considered to be a part of Latrodectus venom, instead potentially being replaced by latrotoxins [14]. However, ICK toxins were recently reported from L. tredecimguttatus [13], and our study revealed that sequences encoding these small peptides were also present among the L. hesperus VSTs. Specifically, four sequences had BLASTx hits of e- 5 or better to members of the spider
CSTX toxin superfamily in UniProt. Each of the predicted proteins from these sequences had 8 cysteine residues, and an ICK scaffold predicted by both Knoter1D and InterProScan, containing three inferred disulfide bonds (Figure 4).

We aligned our four predicted ICK toxin protein sequences (Additional file 4) with 15 sequences retrieved from the ToxProt database [41], representing the range of diversity across the UniProt defined spider CSTX toxin superfamily. The Bayesian and ML trees from this alignment were identical in topology with the exception of two sequences (TXZ10 and TXZ06) that exchanged positions (Figure 5). The L. hesperus sequences form their own strongly supported clade ( $\mathrm{PP}=1.0 ; 100 \%$ bootstrap) within the CSTX superfamily. The most closely related sequences forming a larger clade with the $L$. hesperus ICKs are ICK toxins from three species in the distantly related Superfamily Amaurobioidea [42]. All of these sequences share a conserved 8 cysteine framework [12], but with substantial variation in the number of residues $(8-16)$ between cysteines 6 and 7. Two of the sequences in this clade (Figure 5: omega-ctenitoxin and CpTx1) have demonstrated cytolytic and/or calcium-channel blocking activity [43,44]. Using the four ICK toxin predicted proteins as queries, a BLASTp search of the full $L$. hesperus transcriptome assembly was also performed to identify related sequences lacking venom gland specificity. This search returned a single hit, which appears to be a fragment of an ICK protein that was expressed exclusively in the venom gland, but did not meet the minimum expression level (>1 eCPM) for inclusion in the venom gland specific set.

Small proteins ( $<200$ amino acids) that are comparatively cysteine-rich (at least 6 cysteines, which are necessary to form the three disulfide bonds that are a defining feature of the ICK fold), are potential candidates for novel ICK toxins. Predicted proteins meeting these criteria are in excess in the VSTs (17.2\%) compared to the overall transcriptome $(6.6 \%)$ considering only proteins with an $\mathrm{N}$-terminal methionine. Of the $32 \mathrm{VST}$ sequences that met this definition, 19 did not have a BLAST hit to UniProt at e-5 or better. Seven of the 19 without a BLAST hit had a predicted ICK scaffold from Knoter1D although none had this scaffold predicted by InterProScan (Table 3, Figure 4). Four of the seven were also strongly predicted as toxins (P2 and P3 categories of ClanTox), while one was categorized as possibly toxin-like (P1). These seven proteins ranged in length from 83 to 124 amino acids, possessed 8-10 cysteines (Table 3), and may represent additional instances of distinct ICK toxins in the $L$. hesperus venom-specific transcriptome, for a total of 11 .

\section{CRISP proteins and enzymes}

Two transcripts in the venom gland specific set had BLAST homology to cysteine-rich secretory proteins of 


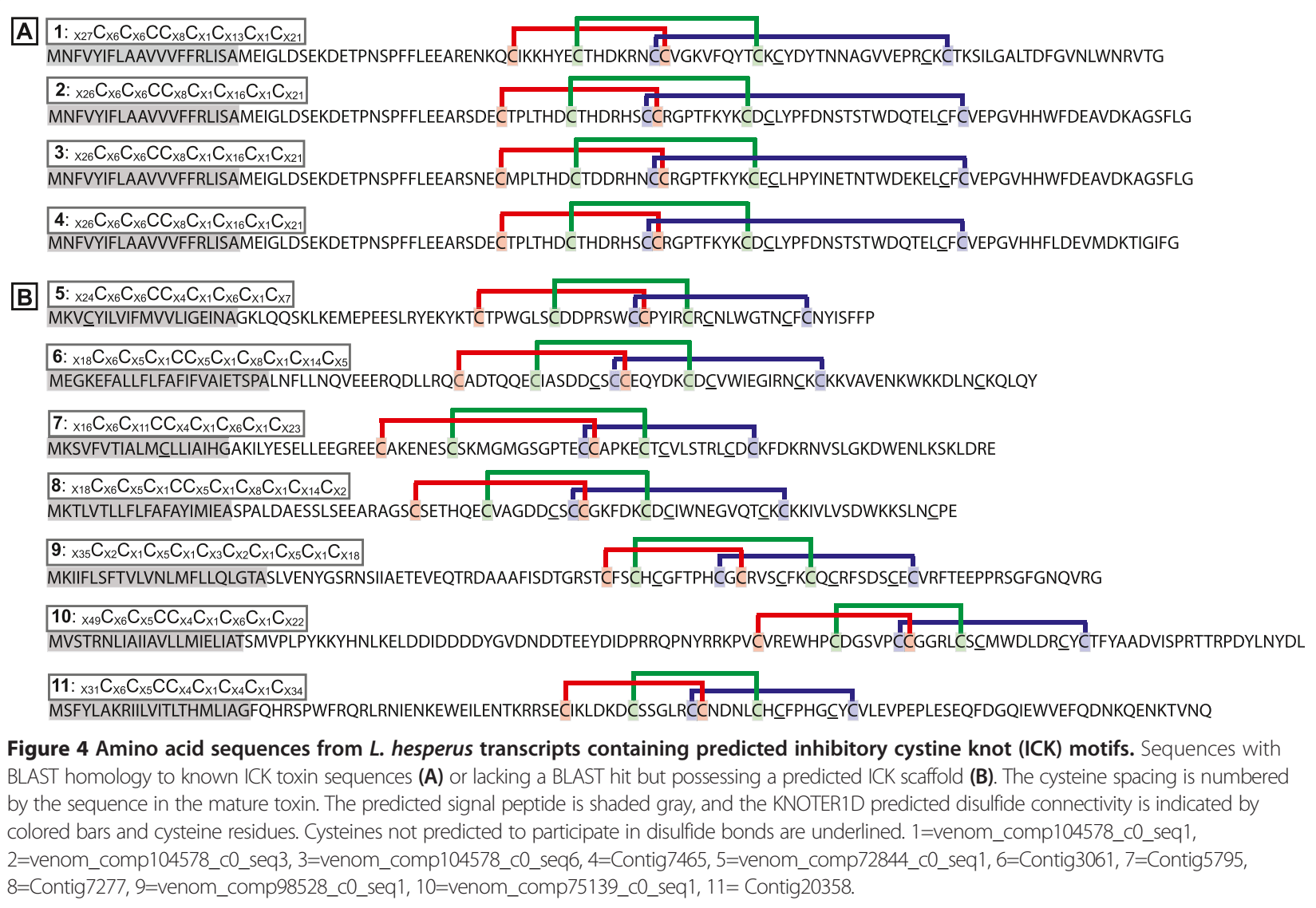

the CRISP family, which are common to the venom of numerous species [1], while a third had a top BLAST hit to an uncharacterized protein, but grouped with the CRISP sequences in clustering analysis (see below). We searched the entire $L$. hesperus transcriptome for other closely related sequences with BLASTp (e-value $\leq \mathrm{e}-20$ ), but lacking in venom gland biased expression. The $L$. hesperus transcriptome did contain three sequences most highly expressed in the cephalothorax or silk glands with BLAST homology to venom gland specific CRISPs, and with UniProt BLASTx hits to CRISP family proteins. We conducted phylogenetic analysis on the alignment of $L$. hesperus CRISP proteins with sequences from a range of venomous and non-venomous invertebrates that have BLAST homology to the L. hesperus CRISPs (Additional file 4). At the largest scale, there were two clades in the Bayesian and ML trees (Figure 6, Additional file 5), which are highly similar with the exception of the placement of a few weakly supported sequences. All sampled spider sequences occur in one clade, together with most other arachnid CRISPs, including those that show evidence of expression in spider or scorpion venom glands. In contrast, some CRISPs with expression in salivary glands of hematophagous ticks are found in the largely arachnid clade, while others appear more closely related to insect
CRISPs (Figure 6). The three $L$. hesperus venom gland specific CRISPs form a highly supported clade with a broadly expressed but closely related $L$. hesperus CRISP. Moreover, a number of scorpion venom gland expressed CRISPs group with a copy from the tick Ixodes scapularis. Other scorpion CRISPs occur in a different clade with all $L$. hesperus CRISPs, whether venom gland specific or not, along with venom gland expressed CRISPs from other spider species.

Transcripts with homology to several types of enzymes were found in the L. hesperus VST set. A total of two hyaluronidases, a single chitinase, and 3 lipases (phospholipase C, AB hydrolase) were identified. A total of 7 distinct serine protease sequences and 8 M13 metalloproteases were found among the 695 in the venom gland specific set. In addition, single sequences with homology to O-sialoglycoprotein endopeptidases and gamma glutamyl transpeptidases were recovered (Additional file 1).

\section{Clustering analysis of venom-gland specific proteins}

We explored the relationships among VSTs using BLASTclust on predicted proteins to identify putative gene families. Under the most stringent clustering criterion (95\% amino acid identity over $95 \%$ of the length of both sequences), 675 of 695 protein sequences did not group with any other sequence, and only 9 multiple transcript clusters 


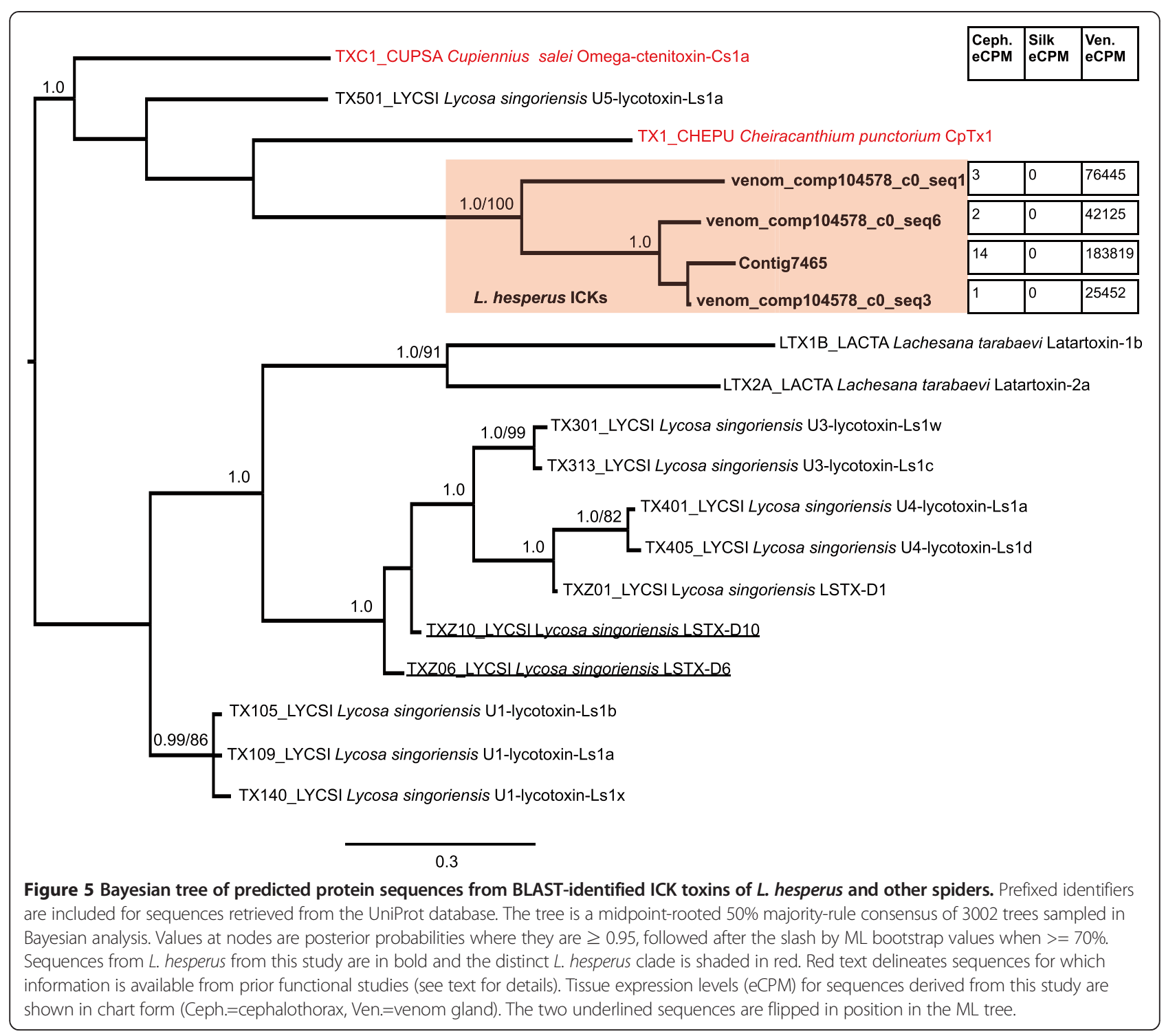

Table 3 Summary of putative toxins with no BLAST hit

\begin{tabular}{|c|c|c|c|c|c|}
\hline ID & Clantox & Knoter1D & Iprscan_domains & \#Cys & Length \\
\hline Venom_comp72844_c0_seq1 & P3 & Knottin & $\mathrm{SP}, \mathrm{NC}$ & 9 & 83 \\
\hline Contig3061 & P2 & Knottin & $\mathrm{SP}, \mathrm{TM}, \mathrm{NC}, \mathrm{CC}$ & 10 & 98 \\
\hline Contig5795 & P3 & Knottin & $\mathrm{SP}, \mathrm{NC}$ & 9 & 95 \\
\hline Contig7277 & P2 & Knottin & $\mathrm{SP}, \mathrm{NC}$ & 10 & 91 \\
\hline Venom_comp98528_c0_seq1 & P1 & Knottin & $\mathrm{SP}, \mathrm{TM}, \mathrm{NC}$ & 10 & 107 \\
\hline Venom_comp75139_c0_seq1 & $\mathrm{N}$ & Knottin & $\mathrm{TM}, \mathrm{NC}, \mathrm{CP}$ & 8 & 124 \\
\hline Contig20358 & N & Knottin & $\mathrm{TM}, \mathrm{NC}, \mathrm{CP}$ & 8 & 116 \\
\hline Venom_Contig10790 & P1 & Negative & None & 6 & 65 \\
\hline Contig2158 & P1 & Negative & None & 5 & 52 \\
\hline Venom_Contig4851 & P1 & Negative & None & 5 & 64 \\
\hline
\end{tabular}

The ID of each sequence is included, together with ClanTox prediction category, Knoter1D prediction of knottin (ICK) scaffold status, protein domains recognized in InterProScan, the number of cysteine residues and protein length. $\mathrm{SP}=$ signal peptide, $\mathrm{TM}=$ transmembrane domain, $\mathrm{NC}=$ non-cytoplasmic domain, $\mathrm{CP}=$ cytoplasmic domain, $\mathrm{CC}=$ coiled-coil. $\mathrm{P} 3=$ =toxin-like, $\mathrm{P} 2=$ probably toxin-like, $\mathrm{P} 1=$ possibly toxin-like, $\mathrm{N}=$ probably not toxin-like. 


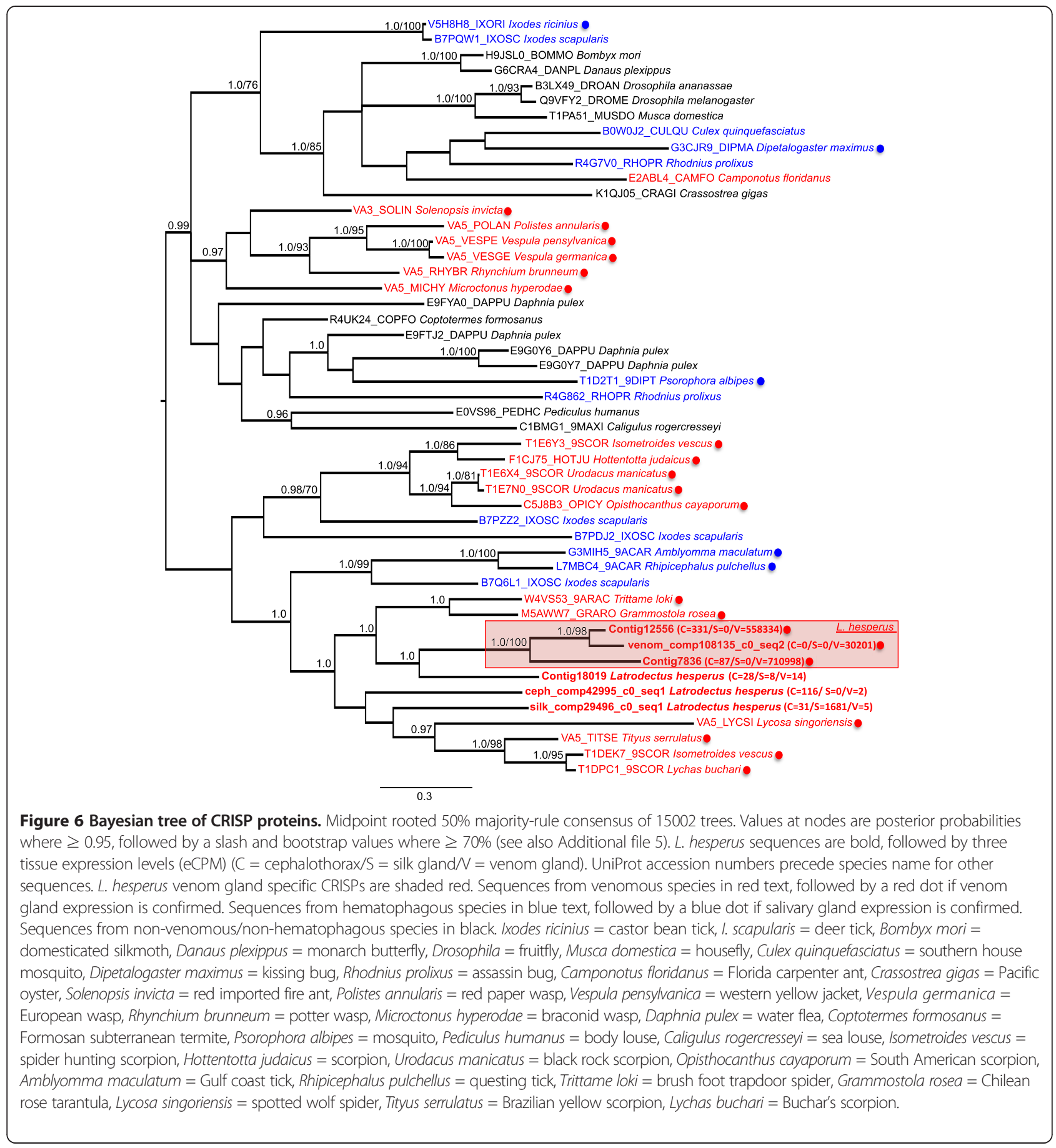

occurred, with the largest containing 3 members. As the sequence identity was reduced while maintaining stringent (95\%) overlap, additional clusters were recovered to a maximum of 20 at $\geq 30 \%$ sequence identity. These 20 clusters contained 48 of 695 transcripts, and the largest cluster included only five sequences. Relaxing the percent overlap of sequences while keeping the $95 \%$ sequence identity threshold produced a similar result, with 44 transcripts in 19 clusters, and a maximum cluster size of five, at $\geq 30 \%$ sequence overlap. When both criteria were relaxed, the number of transcripts in clusters increased rapidly below 65\% sequence identity and $65 \%$ sequence overlap (Figure 7). The most pronounced increase in cluster size (maximum of 34 members) occurred below $40 \%$ overlap and $40 \%$ identity, while the number of clusters decreased slightly. At the most permissive threshold for group formation (30\% overlap, $30 \%$ identity), approximately $22 \%$ of transcripts belonged to 36 groups with at least one other member. 


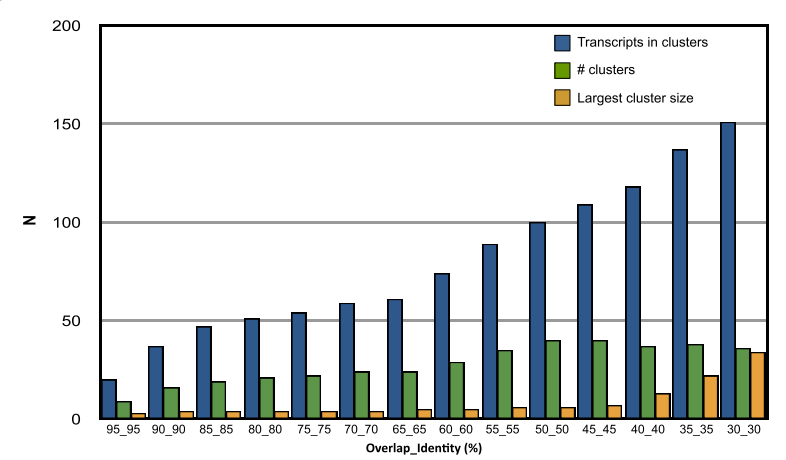

Figure 7 Results of BLAST-based clustering analysis of $L$. hesperus predicted proteins from VSTs. Clustering of sequences was performed across a range of sequence overlap and identity values.

There were several clusters with members homologous to known toxins. Under the most permissive clustering criterion, the largest of these groups had 34 members, all but three of which had best BLASTx hits to latrotoxins in the UniProt database, with the other sequences likely clustered due to weak similarity in the ankyrin repeat regions. A second group contained four additional latrotoxin sequences. Membership in the larger group was highly sensitive to the stringency of the clustering parameters, as at 35\% overlap and 35\% identity, only 22 sequences remained, all with homology to latrotoxins, and at $45 \%$ overlap and $45 \%$ identity this cluster had fragmented into several smaller clusters, the largest of which contained six members (Additional file 6). The four sequences with homology to ICK toxins also formed a group at the lowest clustering stringency, but this group appeared more coherent: these sequences remained clustered as stringency was increased until $75 \%$ overlap at $75 \%$ identity was reached.

Other clusters containing more than five members at the most permissive threshold (30\% overlap, $30 \%$ sequence identity), and representing putative venom gland expressed families, included sequences with homology to cuticular proteins (18 members), M13 metalloproteases (11), leucine-rich repeat (LRR) proteins (7), and serine proteases (6), while the two CRISP proteins identified by BLAST homology clustered with an uncharacterized protein.

\section{Highly expressed venom gland transcripts}

Substantial variation in abundance existed among VSTs (Additional file 1). However, a minority of sequences with BLAST homology to known toxins and associated proteins constituted a significant $(45.8 \%)$ proportion of overall VST expression (Table 1; Figure 8). If the putative ICKs (cysteine-rich small proteins with no BLAST homology but ICK domain predictions) are included this figure rises to $60 \%$. Toxin transcripts were common

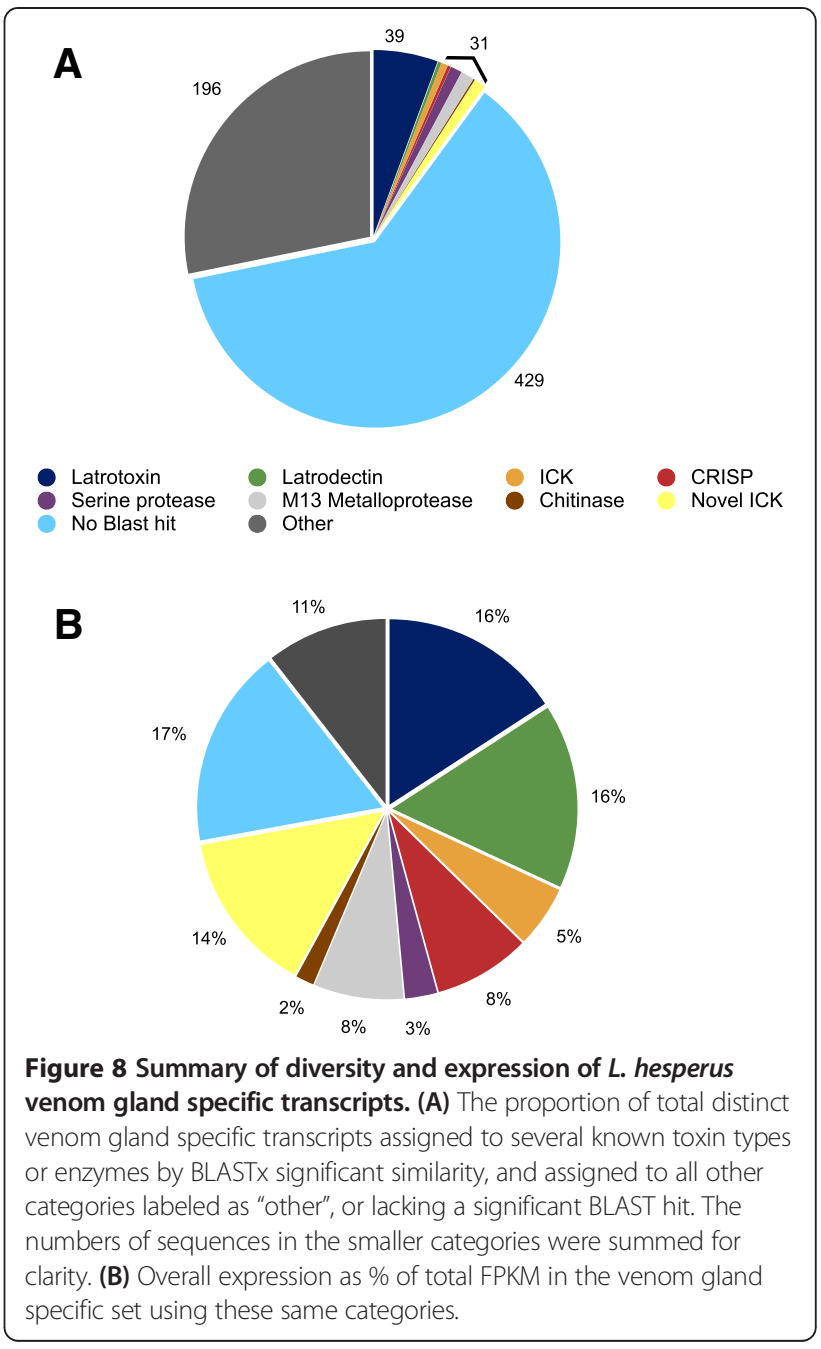

among the most highly expressed VSTs and included sequences with BLASTx homology to known latrotoxins, ICK toxins, CRISP family toxins, and latrodectins (2 of the 3 most highly abundant VSTs). A number of proteases, as well as a chitinase, were amongst the most highly expressed transcripts, and proteases constitute approximately $11 \%$ of the overall expression (Table 1 , Additional file 1). In addition, 4 of 7 small, disulfide-rich proteins with no BLAST hit, but with ICK domain predictions, were amongst the most highly expressed VSTs (Table 3, Additional file 1), as were two other small cysteine-rich proteins with no ICK or ClanTox prediction. While only two distinct latrodectin sequences were found, they constituted approximately $16 \%$ of the total expression for all venom gland specific transcripts (Figure 8). In contrast, the latrotoxins contribute a similar proportion of transcript abundance (15.8\%) but are much richer in sequence diversity, with 39 sequences that likely represent at least 20 paralogs (Figure 8). 


\section{Proteomic and bioinformatic analysis of secreted components}

Using Multidimensional Protein Identification Technology (MudPIT) analyses, we identified 61 proteins from an L. hesperus protein database that matched peptides collected from $L$. hesperus venom with mass spectrometry techniques (Additional file 7). The analyzed protein database contained 105,520 L. hesperus proteins predicted from two transcript sets: (1) 103,635 from the overall L. hesperus transcriptome de novo assembly, and (2) 483 venom gland ESTs, together with $414 \mathrm{~L}$. hesperus proteins from NCBI's $n r$ database. The venom detected proteins included 21 latrotoxins, 1 ICK toxin, and 6 CRISP family toxin proteins (Table 4). Four other sequences from the cysteine-rich set of unknown proteins were also found in the venom, including two that potentially represent additional ICK toxins (Table 3, Additional file 7). Several types of enzymes were identified in $L$. hesperus venom, including hyaluronidases, chitinase, serine proteases and metalloproteases, as were several leucine-rich repeat proteins and three latrodectins (Table 1; Additional file 7). Of the proteins matched, most (49) were predicted from VSTs, while 3 were from transcripts that were venom gland biased, but were not in the upper $2.5 \%$ of the distribution of eCPM ratio values. Eight protein sequences predicted from venom gland ESTs, and one from a previous study of latrotoxins [25], were also detected in L. hesperus venom.

Approximately $12.5 \%$ (87) of protein translations from the $L$. hesperus VSTs possessed a predicted signal sequence. If only the 313 proteins with a putative methionine start

Table 4 Predicted neurotoxin proteins identified in venom

\begin{tabular}{|c|c|c|c|}
\hline ID & Best BLAST hit & \# unique peptides & $\%$ coverage \\
\hline Contig14 & CRISP & 42 & $51.90 \%$ \\
\hline Contig29 & CRISP & 5 & $6.87 \%$ \\
\hline LHV_229_Sp6 & CRISP & 5 & $24.30 \%$ \\
\hline LHV_312_Sp6 & CRISP & 2 & $3.77 \%$ \\
\hline Contig12556 & CRISP & 21 & $38.90 \%$ \\
\hline Contig7836 & CRISP & 25 & $44.30 \%$ \\
\hline venom_comp104578_c0_seq1 & ICK & 2 & $27.00 \%$ \\
\hline HV209_Sp6_4 & a-latrotoxin & 2 & $11.60 \%$ \\
\hline gi|380876959|sp|PODJE7.1|LITD_LATHE & $\delta$-latroinsectotoxin & 3 & $17.00 \%$ \\
\hline Contig2826 & a-latroinsectotoxin & 47 & $35.40 \%$ \\
\hline Contig9724 & a-latrotoxin & 23 & $23.50 \%$ \\
\hline Contig9749 & a-latrotoxin & 43 & $40.30 \%$ \\
\hline Venom_comp106397_c0_seq1 & $\delta$-latroinsectotoxin & 32 & $30.50 \%$ \\
\hline Venom_comp107842_c0_seq1 & $\delta$-latroinsectotoxin & 15 & $30.00 \%$ \\
\hline Venom_comp107842_c2_seq1 & $\delta$-latroinsectotoxin & 3 & $37.70 \%$ \\
\hline Venom_comp109612_c1_seq1 & $\delta$-latroinsectotoxin & 4 & $19.30 \%$ \\
\hline Venom_comp110241_c0_seq1 & a-latrotoxin & 7 & $8.98 \%$ \\
\hline Venom_comp110271_c0_seq3 & a-latrotoxin & 2 & $6.88 \%$ \\
\hline Venom_comp110271_c1_seq1 & a-latrotoxin & 28 & $28.00 \%$ \\
\hline Venom_comp110278_c1_seq1 & a-latrotoxin & 25 & $26.60 \%$ \\
\hline Venom_comp110315_c0_seq1 & a-latrotoxin & 8 & $25.80 \%$ \\
\hline Venom_comp110315_c1_seq1 & a-latrotoxin & 14 & $20.20 \%$ \\
\hline Venom_comp110343_c2_seq1 & $\delta$-latroinsectotoxin & 3 & $5.50 \%$ \\
\hline Venom_Contig10080 & $\delta$-latroinsectotoxin & 8 & $8.76 \%$ \\
\hline Venom_Contig10081 & $\delta$-latroinsectotoxin & 4 & $4.40 \%$ \\
\hline Venom_Contig10082 & $\delta$-latroinsectotoxin & 4 & $4.42 \%$ \\
\hline Venom_Contig10158 & $\delta$-latroinsectotoxin & 6 & $13.30 \%$ \\
\hline Venom_Contig11531 & a-latrocrustotoxin & 44 & $35.90 \%$ \\
\hline
\end{tabular}

${ }^{a}$ Contains EST sequences LHV_167_Sp6, LHV_012_T7, LHV_012_Sp6, LHV_194_Sp6, LHV_167_T7.

${ }^{\mathrm{b}}$ Contains EST sequences LHV_250_Sp6, LHV_144_Sp6, LHV_069_Sp6, LHV_069_T7, LHV_511_Sp6, LHV_123_T7, LHV_123_Sp6, LHV_144_T7, LHV_457_Sp6. The type of toxin identified by the top BLAST hit to each transcript is shown in column 2. Proteomic evidence is displayed in columns 3 (unique peptides mapping to sequence) and 4 (\% coverage of predicted protein with mapped peptides). 
codon are considered, this figure rises to $24.9 \%$. Amongst the toxin homologs in this set, none of the predicted latrotoxin proteins contained a typical eukaryotic signal sequence, while four of four ICK toxins, both CRISP toxins, and both latrodectins, contained a signal sequence, as did all seven other potential ICK toxins with no significant BLAST homology. Five proteases (four serine proteases and one metalloprotease) also had a predicted signal sequence. Thirty-six of the 49 predicted proteins from VSTs detected in venom by mass spectrometry contained an Mstart, of which 22 (61\%) had predicted signal sequences, consistent with their function as a venom component, as opposed to having an intracellular function.

\section{Discussion}

Spiders are the most species-rich clade of venomous metazoans, and it is likely that millions of toxic compounds remain to be identified in their venom $[7,45]$. Next generation transcriptomic and proteomic methods, when used in combination, offer a powerful approach to cataloguing and understanding this complexity, as well as its evolution. By applying these methods to Latrodectus hesperus, in the context of a multi-tissue expression analysis, we have identified 695 transcript sequences with strongly biased venom gland expression in this species and confirmed the presence of 61 proteins in its venom. The inferred functions of these sequences indicate that the venom of black widow spiders is extremely diverse at the molecular level, and is the product of a complex evolutionary history.

\section{Molecular diversity in the $L$. hesperus venom gland and functional implications}

We found that only $22 \%$ of the $695 \mathrm{~L}$. hesperus VSTs shared some sequence overlap at the protein level through BLASTclust analyses, implying that a wide diversity of proteins contribute to venom gland function. Nevertheless, we estimated that at least 20 distinct latrotoxin paralogs are expressed in the black widow venom gland, constituting by far the largest gene family in the venom gland specific set of sequences. The latrotoxin proteins predicted from these transcripts were divergent in amino acid sequence and motif organization (Figure 2, Figure 3), and thus it is likely that they represent distinct loci. While seven latrotoxins have been assigned names based on their taxonomic specificity (5 insect-specific, 1 vertebrate-specific, 1 crustaceanspecific) in the related species $L$. tredecimguttatus [38], the sequence of only four of these seven functionally characterized latrotoxins are definitively known [19-22]. We identified orthologs of these four functionally characterized latrotoxins in our transcriptome, but have also quintupled the number of sequenced latrotoxin paralogs in L. hesperus. While the functionality of these novel latrotoxins is unknown, some of these sequences have best BLASTx hits to the vertebrate-specific $\alpha$-latrotoxin. Although functional testing is a requirement for confirmation, some of these sequences could represent heretofore unknown vertebrate specific neurotoxins. Such discoveries are significant because vertebrate neurotoxins have important applications in neurophysiological research, considering the fundamental role of $\alpha$-latrotoxin in deciphering the molecular mechanisms of neurotransmission. The extensive diversity found among the vertebrate receptors of latrotoxins such as neurexins and latrophilins [46-48], suggests that some of these new latrotoxin variants may interact specifically with different receptor isoforms and could play important roles in their characterization. The variable number of ankyrin domains predicted from nearly full-length sequences in this study could contribute to altered functionality, including the ability of latrotoxin monomers to tetramerize, given the role of ankyrin repeats in protein-protein interactions [39].

Small cysteine rich neurotoxic proteins with the inhibitor cystine knot motif dominate the venoms of many spider species [11]. Our BLAST analyses identified four putative ICK toxin sequences amongst the $L$. hesperus VSTs and one was present in the exuded venom. In addition to these ICK toxins, other small cysteine-rich sequences were venom gland specific in expression and some were present in the venom. Some of these toxins may also be ICK toxins as they possess a predicted ICK domain, while others may represent distinctly different molecular scaffolds, although further research is necessary on their structure and function. The presence of both latrotoxins and ICK toxins in Latrodectus venom also suggests novel avenues in research as to how small, selective ion-channel toxins may act synergistically with the non-selective cation channels created by latrotoxin pores in the presynaptic membrane [49,50]. Three additional cysteine-rich proteins with homology to CRISP toxins (or found by clustering analyses) were also strongly biased towards expression in $L$. hesperus venom gland and present in the venom. CRISP family members were also found to be expressed in the venom gland of the related species $L$. tredecimguttatus [13], indicating that this toxin type may be more widespread within the genus.

Among the other venom gland specific transcripts were multiple sequences with homology to proteins with nervous system related functions (Additional file 1). Examples of these included bruchpilot from Drosophila melanogaster, involved in synaptic plasticity and regulation [51] and neural cell adhesion molecule L1, the Drosophila ortholog of which plays a critical role in neural development [52]. L1-type cell adhesion molecules also play a role in presynaptic organization, and often interact with ankyrin repeat containing proteins [53]. Given the importance of the ankyrin repeat-containing latrotoxins in black widow venom, the venom gland biased expression of these transcripts is intriguing, although their links to the action of latrotoxins are speculative at this point. Lastly, eight 
sequences with homology to leucine-rich repeat (LRR) proteins were also venom gland specific, and a number of these proteins play key roles in neuronal development and maintenance in both invertebrates and vertebrates $[54,55]$. These results suggest that homologs of spider proteins involved in neuronal development or function are being coopted for venom expression, or the potential for molecular mimicry of neuronal proteins by unrelated venom gland expressed sequences.

\section{Evolutionary diversification of black widow venom toxins}

The development of pools of diverse toxin molecules in venom often involves the expansion of gene families [7]. This process can generate large numbers of distinct transcripts and peptides in certain toxin classes. In cone snails, species may produce from 100-300 small ICK peptides known as conotoxins [9]. Conotoxins are notable for their rapid evolution and the extreme divergence among paralogs within a species at the amino acid level [56]. Similarly, sequencing of spider venom gland transcripts has revealed single species ICK toxin libraries containing more than 100 distinct members $[12,57]$. While ICK toxin sequences can also differ dramatically among spiders, clades of more closely related sequences also occur in some spider species, and likely represent more recent, species-specific gene family diversification [45]. This may be true in the case of the L. hesperus sequences with BLAST homology to known ICKs. Yet, we also found seven additional ICK motif containing sequences, which were more diverse in length, signal sequence and cysteine arrangement, suggesting the recruitment of multiple ICK motif encoding proteins for black widow venom expression.

Latrotoxins, while the most diverse toxin type in this study, as a whole appear to be limited in phylogenetic distribution, and the origins of these toxins are obscure. Only one paralog ( $\alpha$-latrotoxin) has been recognized outside the genus Latrodectus, and to date latrotoxins are only known from three genera of Theridiidae [26]. Although repeated ankyrin domains are found in a wide range of unrelated proteins of various functions [58], the latrotoxin $\mathrm{N}$-terminal region appears to be somewhat unique to latrotoxins. A BLASTp search with latrotoxin Nterminal sequences (first 320 amino acids) against the nonvenom gland specific $L$. hesperus transcriptome did not find any significant hits. However, we performed a BLASTp search with the $L$. hesperus $\alpha$-latrotoxin $\mathrm{N}$-terminal region against NCBI's $n r$ database, and found a significant hit to a hypothetical protein from Diplorickettsia massiliensis (Accession WP_010598965; e-score 1e-16), an obligate intracellular bacteria isolated from the tick Ixodes ricinus, which is a human disease vector. In addition to N-terminal region sequence similarity, the overall length (1286 amino acids) and possession of multiple ankyrin repeats of this bacterial protein are reminiscent of latrotoxins. A recent study by Zhang et al. [59] described similarities between the C-terminal domain of latrotoxins and proteins from arthropod bacterial endosymbionts such as Wolbachia and Rickettsiella, and suggested that spider latrotoxins were acquired via lateral gene transfer from bacteria. Alternatively, Garb and Hayashi [26] suggested a possible link between latrotoxins and dTRP1a, a Drosophila calcium permeable transmembrane channel protein involved in sensitivity to temperature and chemical irritation that contains numerous ankyrin repeats. As genome sequences for Latrodectus and related theridiid species become available, these questions regarding the evolutionary origin of latrotoxins may become answerable.

Given the broader phylogenetic distribution of $\alpha$ latrotoxin outside of $L$. hesperus [26], it will be important to determine if the additional latrotoxins we uncovered have orthologs in closely related species having venom that is less toxic to vertebrates when compared to venom from black widows. Phylogenetic analyses of the latrotoxin family across multiple species may illuminate the ecological adaptations of widow spiders, particularly in terms of understanding the functional utility of latrotoxins for a generalist predator of diverse insects and small vertebrates. Three insect specific latrotoxins previously identified in protein separation studies [38] may be represented in the additional latrotoxins we have recovered, but the functional and taxonomic specificity of the others remains to be determined. Such functional analyses will be necessary to reconstruct whether ancestral latrotoxins have undergone a functional shift from arthropod to vertebrate specificity or vice versa. A comprehensive latrotoxin phylogeny across species could also determine whether gene family expansions are lineage-specific, and correlate with increased venom toxicity and diet breadth.

In contrast to latrotoxins and ICK toxins, the cysteine-rich secretory proteins (CRISPs) are not particularly diverse within the $L$. hesperus VSTs, but we were able to identify three additional transcripts with homology to CRISPs that do not show venom gland specificity. A CRISP phylogeny including diverse venomous, non-venomous and hematophagous arthropods indicates a dynamic evolutionary history for this gene family, with multiple recruitments to function in venom or salivary glands, including a potentially recent CRISP protein recruitment for venom function in Latrodectus. A similar conclusion was reached with a less densely sampled, but broader taxonomic selection of CRISPs [1], and more extensive arthropod transcriptomic and genomic resources may identify the gene duplications and changes in tissuespecific expression patterns leading to this pattern.

\section{Highly expressed transcripts, venom composition and secretory mechanisms}

Among the venom gland specific transcript set, overall expression is dominated by putative neurotoxins and their 
associated molecules, although they make up only a minority of the distinct transcripts. Strikingly, the proportion of transcripts that latrodectins represent is similar to that for all latrotoxin sequences, although latrodectin sequence diversity was at least ten times lower than that of latrotoxins. This suggests that the role of latrodectins in facilitating latrotoxin toxicity may be the same for all latrotoxins, including novel forms identified in this study. Protease expression also accounts for a substantial proportion of VST abundance, and several proteases were amongst the most abundant transcripts in the venom gland specific set.

Proteomic analysis of $L$. hesperus venom also indicates that at least some proteases are secreted, as together with other enzymes (hyaluronidases and chitinase), they were identified in $L$. hesperus venom. Hyaluronidases are found in venom from a range of spider species [14], but whether proteases are an active component of venom in spiders has been a subject of some debate, as some authors argue that protease activity in venom is due to digestive secretion contamination [60]. Our finding of proteases with venom gland specificity, together with the presence of a subset of proteases in the venom, some with predicted secretory signal sequences, may be related to a dual function. Some $L$. hesperus proteases may in fact function in prey immobilization, either acting as toxin spreading factors, or in hemostasis disruption, as is the case in snakes $[7,61]$, while others may be involved in processing toxin preproproteins into mature toxins [25].

Our mass spectrometry analyses indicated that the majority of the neurotoxin transcripts specific to the venom gland encoded peptides and proteins that were secreted into the venom. Predicted neurotoxins that were not present in collected venom may reflect the variability inherent in venom-related gene expression, as data acquisition for the transcriptome and proteome was performed on different individuals. It may also reflect variation in the processes of translation or secretion among individual spiders. Overall, the limited number of venom gland specific genes whose products are found in the venom itself is rather unexpected, given the purported mechanism of $L$. hesperus secretion into the venom gland lumen, in which the secretory cells disintegrate and expel the entirety of their contents [23,62]. Yet there would appear to be some filtering mechanism that is selective against most proteins from VSTs, as few appear in the venom itself. The possession of a signal sequence may constitute such a filter. While only a minority (25\%) of complete predicted proteins from VSTs have a predicted signal sequence, the majority of proteins (67\%) identified in the venom by mass spectrometry have predicted signals. Latrotoxins seem to be an exception, lacking a typical eukaryotic secretion signal, yet being common in the venom itself. However, previous work has indicated the presence of a cleaved sequence on the $\mathrm{N}$-terminus that could potentially function as a non-canonical secretory signal [21].

\section{Conclusions}

In this study, next-generation RNA sequencing of multiple tissues coupled to proteomics has provided a wealth of insight into venom gland expression and the molecular complexity of Latrodectus venom. Numerous new variants of known toxins were identified, and potentially novel toxins of unknown function recovered, suggesting the need for a fundamental reconsideration of the functional activities of black widow spider venom in natural prey and in human envenomation. The extreme pain associated with black widow spider bites is typically accompanied by additional symptoms (e.g., diaphoresis, hypertension, paresthesia, fasiculations [63]), which in addition to $\alpha$ latrotoxin, may be caused by other toxins uncovered in this study. This expanded toxin library can also be mined for novel molecular probes or drug leads. Of particular interest for neurophysiology is the large number $(\geq 20)$ of previously unknown latrotoxin variants and 11 ICK motif containing proteins discovered in this study, which may offer new avenues for dissecting the molecular mechanism of neurotransmitter release and for characterizing neuronal ion channels. These functionally diverse latrotoxins comprise a large venom gland expressed gene family with a highly restricted phylogenetic distribution, suggesting they have undergone a rapid evolutionary expansion in black widow spiders.

\section{Methods}

\section{L. hesperus transcriptome sequencing and assembly}

Paired-end Illumina sequencing was performed by the Genomics Core at the University of California, Riverside, on cDNA libraries generated using the Illumina mRNA sequencing sample preparation kit with mRNA from three tissue types: (1) venom gland, (2) silk glands and (3) cephalothorax minus venom glands, each in a single lane [37]. After trimming of adapters and low quality sequence, reads from each individual library were separately assembled using Trinity [64], and subjected to CAP3 [36] to merge transcripts under default parameters and reduce redundancy in the transcript set, producing contigs with the tissue type as a prefix (i.e. venom_Contig0000). CAP3 was then applied a second time to merge transcripts across tissue-specific assemblies and produce a set of contigs with no prefix (i.e. Contig0000) as well as retaining contigs from the tissue specific CAP3 assemblies with a tissuespecific prefix, together with non-merged transcripts that retain the original Trinity nomenclature (i.e. venom_comp00000_c0_seq0) with a prefix indicating their tissue origin [65]. All sequences were screened for homology to the UniProt database using BLASTx with an e-value cutoff of $1 \mathrm{e}-5$. Open reading frames (ORFs) for all transcripts were predicted in all six frames using GetORF, filtering out ORFs less than $90 \mathrm{bp}$ in length. A best protein prediction for each contig was generated with a custom Perl 
script by (1) extracting the longest reading frame in the same frame as the best BLASTx hit, or (2) by extracting the longest reading frame for contigs lacking a BLASTx hit. However, proteins with a methionine start codon were selected if bounded by stop codons on the $5^{\prime}$ and 3 ' ends, indicating the potential for a full-length ORF, and if the M-start ORF was at least $75 \%$ of the longest predicted ORF.

After CAP3 assembly at the nucleotide level some transcripts that produced identical amino acid sequences persisted in the data set. Hence we further filtered the transcript set to produce a non-redundant set of proteins and their associated nucleotide sequences. BLASTclust [66] was employed to identify sets of protein sequences in which members were identical over their entire region of overlap. In cases in which proteins varied in length within a cluster, all but the longest member of the cluster was removed from both the protein and nucleotide sequence libraries using a custom Perl script. Otherwise, the first member was arbitrarily chosen to represent that cluster.

\section{Identification of venom gland specific transcripts}

To identify venom gland specific transcripts (VSTs), RSEM [67] was used to estimate transcript abundances by mapping reads from the venom, cephalothorax and silk libraries against the assembled and filtered non-redundant transcriptome using Bowtie with default parameters [68]. Expected read counts per million (eCPM) in each tissue for each transcript were calculated and the distribution of the $\log$ of the ratio of eCPM of venom gland to silk and venom gland to cephalothorax for each transcript was plotted. Transcripts for which venom gland expression of greater than one eCPM was observed, with zero eCPM in the other two tissues, were identified. Further VSTs were identified as those with a ratio of venom eCPM/silk eCPM and venom eCPM/cephalothorax eCPM in the upper $2.5 \%$ of the distribution of the remaining transcripts, and at least one eCPM in venom. Together, transcripts from these two categories constitute the venom gland specific set. Fragments per kilobase per million reads (FPKM) values were also calculated in RSEM for comparing abundances amongst VSTs.

\section{Functional analysis of venom gland specific transcripts}

GO terms were retrieved from UniProt-GOA for the best BLASTx hit to each sequence and used to annotate the $L$. hesperus sequence set. Additional GO terms were mapped by searching the Pfam-A database for sequence homology to predicted protein sequences using the probabilistic Hidden Markov models implemented in HMMER 3.0 [69].

To correct for potential transcript length bias in differential expression in RNA-Seq experiments, GOseq [70] was performed to find overrepresented gene ontology categories in the set of venom gland specific transcripts to identify biological processes and functions important in the venom gland. This method corrects for the violation of the assumption that all genes are equally likely to be identified as differentially expressed, an assumption that does not hold for read count based methods such as RNA-Seq, and the violation of which causes false positives for categories with an excess of long genes in GO overrepresentation analysis.

\section{Identification of toxins in the venom gland specific set}

Sequences with homology to known toxins were identified in the UniProt BLASTx results using text searches. We identified the potential presence of families of toxin and other transcripts specifically expressed in the venom gland of $L$. hesperus by clustering predicted protein sequences using the BLASTclust algorithm under both permissive and stringent criteria. The BLASTclust output was parsed with a custom Perl script to calculate group sizes, group numbers and group composition by appending BLASTx results.

InterProScan [71] was used on predicted proteins to identify the domain architecture of gene products. ClanTox [72] was used to predict the potential toxicity of translated proteins. The algorithm used takes into account features of the frequency and distribution of cysteine residues in the primary sequence from known peptide toxins [73]. ClanTox produces four categories of toxin predictions based on statistical confidence ranging from $\mathrm{N}=$ probably not toxinlike to P3 = toxin-like. Knoter1D was used to predict the connectivity of inhibitor cystine knot structures (also referred to as knottins) from the primary sequence of peptides and proteins [74]. Given that toxins function within an extracellular secretion, predicted proteins were scanned for the presence of a signal sequence indicating targeting to the secretory pathway using SignalP 4.1 [75].

\section{Venom collection and mass spectrometry}

We determined the proteins present in the venom of $L$. hesperus by collecting venom exuded by anesthetized adult females subject to electrostimulation with a $10 \mathrm{~V}$ current via a capillary tube, and subsequently diluting the venom in $5 \mu \mathrm{L}$ of distilled water. The trypsin-digested diluted venom was analyzed by MudPIT analysis [76], performed by the Arizona Proteomics Consortium at the University of Arizona. This method uses a multidimensional liquid chromatography separation followed by tandem mass spectrometry (LC-MS/MS) and the Sequest algorithm [77] to identify digested peptides in L. hesperus venom secretions. Scaffold software (Proteome Software, Portland, Oregon) was then used to map peptides found in venom to the predicted protein sequences from the $L$. hesperus assembled transcriptome, together with $L$. hesperus venom gland ESTs, and all $L$. hesperus protein sequences available at NCBI, to identify secreted products. 
Only sequences with protein and peptide probabilities in excess of $95 \%$, and with at least two mapped unique peptides were considered as present in venom.

\section{Phylogenetic analysis}

Alignments of amino acid sequences were constructed with the COBALT [78] web server at NCBI using default settings for gap penalties and query clustering, and with RPS BLAST enabled. Alignments were trimmed manually or with trimAl 1.2 [79] using the automated1 setting to remove regions with an excessive amount of missing data or poorly aligned regions. Phylogenetic trees were constructed for members of specific gene families using Bayesian analysis of amino acid sequences in Mr. Bayes 3.2.2 [80] sampling across fixed amino acid rate matrices. Two simultaneous runs of 1,000,000-5,000,000 generations using a single Markov chain were performed. Convergence was achieved in all analyses as determined by an average standard deviation of split frequencies $<0.01$, effective sample sizes for all parameters $>100$, and potential scale reduction factors for all parameters of approximately 1 . The first $25 \%$ of trees sampled were discarded as burn-in and a 50\% majority rule consensus was constructed for each analysis using posterior probability (PP) as a measure of clade support. Maximumlikelihood trees for the same set of gene families were found using RAxML [81] using the BLOSUM62 substitution rate matrix with gamma distributed rate variation among sites. 1000 bootstrap pseudoreplicates were performed to assess support for clades.

\section{Availability of supporting data}

All reads and the final transcriptome described in the manuscript are available under BioProject accession PRJNA242358. Illumina sequence reads have been deposited at NCBI's SRA archive under the following numbers (Venom: Sample: SAMN2720862, Experiment: SRX512000, Reads: SRR1219652; Cephalothorax: Sample: SAMN2708870, Experiment: SRX511999, Reads: SRR1219650; Silk: Sample: SAMN2720861, Experiment: SRX512001, Reads: SRR12 19665). Venom gland ESTs are available under NCBI accession numbers JZ577614-JZ578096 [82].

\section{Additional files}

Additional file 1: A supplemental table that contains expression as FPKM for all transcripts in the venom gland specific set together with top BLAST hit for identification, and expected counts per million (eCPM) for each transcript across the three tissues.

Additional file 2: A supplemental table showing a summary of the representation of protein domains identified by InterProScan from four major databases (SMART, Pfam, ProSite, PRINTS).

Additional file 3: A supplemental table that contains all InterProScan predictions on the set of 695 venom gland specific proteins.

Additional file 4: COBALT alignments of ICK and CRISP proteins.
Additional file 5: Maximum-likelihood tree of CRISP proteins.

Additional file 6: A supplemental table that contains detailed results of cluster membership at each level of stringency indicated in Figure 7.

Additional file 7: A supplemental table that shows the results of proteomic analysis, including all predicted proteins from transcriptomic and previously published data that were identified in venom by MudPIT analysis, and salient information about the protein, including whether it had a methionine start and predicted signal peptide.

\section{Abbreviations}

RNA-seq: RNA sequencing; VST: Venom gland specific transcript; kDa: Kilodaltons; ICK: Inhibitor cystine knot; CRISP: Cysteine-rich secretory protein; eCPM: Expected counts per million; MudPIT: Multidimensional protein identification technology; EST: Expressed sequence tag; CDNA: Complementary DNA; ORF: Open reading frame; FPKM: Fragments per kilobase per million reads; GO: Gene ontology.

\section{Competing interests}

The authors declare that they have no competing interests.

\section{Authors' contributions}

$\mathrm{RH}$ analyzed the data and drafted the manuscript. NA conceived the experiments, generated data, and analyzed the data. TC analyzed the data. $\mathrm{CH}$ conceived the experiments and generated data. JG conceived the experiments, generated data, analyzed the data and drafted the manuscript. All authors edited and approved the final manuscript.

\section{Acknowledgments}

We appreciate the analytical and intellectual contributions of Alex Lancaster, Susan Corbett, Ryan Fitzpatrick, Peter Arensburger and George Tsaprailis towards completing this manuscript. This work was supported by the National Institutes of Health (1F32GM083661-01 and 1R15GM097714-01 to JG; F32 GM78875-1A to NA), and National Science Foundation (IOS-0951886 to NA, IOS-0951061 to CH).

\section{Author details}

'Department of Biological Sciences, University of Massachusetts, Lowell, MA 01854, USA. Department of Biology, Washington and Lee University, Lexington, VA 24450, USA. ${ }^{3}$ Department of Biology, University of California, Riverside, CA 92521, USA.

Received: 30 December 2013 Accepted: 8 May 2014

Published: 11 June 2014

\section{References}

1. Fry BG, Roelants $K$, Champagne DE, Scheib H, Tyndall JDA, King GF, Nevalainen TJ, Norman JA, Lewis RJ, Norton RS, Renjifo C, de la Vega RCR: The toxicogenomic multiverse: convergent recruitment of proteins into animal venoms. Annu Rev Genomics Hum Genet 2009, 10:483-511.

2. Rash LD, Hodgson WC: Pharmacology and biochemistry of spider venoms. Toxicon 2002, 40:225-254.

3. Casewell NR, Wüster W, Vonk FJ, Harrison RA, Fry BG: Complex cocktails: the evolutionary novelty of venoms. Trends Ecol Evol 2013, 28:219-229.

4. Platnick NI: The World Spider Catalog, Version 14.0. [http://research.amnh. org/entomology/spiders/catalog/index.html]

5. King GF: The wonderful world of spiders: preface to the special Toxicon issue on spider venoms. Toxicon 2004, 43:471-475.

6. Escoubas P, Sollod B, King GF: Venom landscapes: mining the complexity of spider venoms via a combined cDNA and mass spectrometric approach. Toxicon 2006, 47:650-663.

7. King GF, Hardy MC: Spider-venom peptides: structure, pharmacology, and potential for control of insect pests. Annu Rev Entomol 2013, 58:475-496.

8. Possani L: Peptides and genes coding for scorpion toxins that affect ion-channels. Biochimie 2000, 82:861-868.

9. Olivera BM, Cruz LJ: Conotoxins, in retrospect. Toxicon 2001, 39:7-14.

10. Isbister GK, White J: Clinical consequences of spider bites: recent advances in our understanding. Toxicon 2004, 43:477-492.

11. Vassilevski AA, Kozlov SA, Grishin EV: Molecular diversity of spider venom. Biochemistry (Moscow) 2009, 74:1505-1534 
12. Zhang $Y$, Chen J, Tang X, Wang F, Jiang L, Xiong X, Wang M, Rong M, Liu Z, Liang S: Transcriptome analysis of the venom glands of the Chinese wolf spider Lycosa singoriensis. Zoology 2010, 113:10-18.

13. He Q, Duan Z, Yu Y, Liu Z, Liu Z, Liang S: The venom gland transcriptome of Latrodectus tredecimguttatus revealed by deep sequencing and cDNA library analysis. PLOS ONE 2013, 8:e81357.

14. Kuhn-Nentwig L, Stöcklin R, Nentwig W: Venom composition and strategies in spiders. In Adv Insect Physiol, Volume 40. London: Elsevier; 2011:1-86.

15. Saez NJ, Senff S, Jensen JE, Er SY, Herzig V, Rash LD, King GF: Spider-venom peptides as therapeutics. Toxins 2010, 2:2851-2871.

16. Adams ME, Herold EE, Venema VJ: Two classes of channel-specific toxins from funnel web spider venom. J Comp Physiol A 1989, 164:333-342.

17. Wullschleger B: Spider venom: enhancement of venom efficacy mediated by different synergistic strategies in Cupiennius salei. J Exp Biol 2005 208:2115-2121.

18. Olivera BM: E.E. Just Lecture, 1996. Conus venom peptides, receptor and ion channel targets, and drug design: 50 million years of neuropharmacology. Mol Biol Cell 1997, 8:2101-2109.

19. Kiyatkin NI, Dulubova IE, Chekhovskaya IA, Grishin EV: Cloning and structure of cDNA encoding a-latrotoxin from black widow spider venom. FEBS Lett 1990, 270:127-131.

20. Kiyatkin N, Dulubova I, Grishin E: Cloning and structural analysis of alphalatroinsectotoxin cDNA. Abundance of ankyrin-like repeats. Eur J Biochem 1993, 213:121-127.

21. Dulubova IE, Krasnoperov VG, Khvotchev MV, Pluzhnikov KA, Volkova TM, Grishin EV, Vais H, Bell DR, Usherwood PN: Cloning and structure of deltalatroinsectotoxin, a novel insect-specific member of the latrotoxin family: functional expression requires C-terminal truncation. J Biol Chem 1996, 271:7535-7543.

22. Danilevich VN, Luk'ianov SA, Grishin EV: Cloning and structure of gene encoded alpha-latrocrustotoxin from the black widow spider venom. Bioorg Khim 1999, 25:537-547.

23. Ushkaryov Y: a-Latrotoxin: from structure to some functions. Toxicon 2002, 40:1-5.

24. Rohou A, Nield J, Ushkaryov YA: Insecticidal toxins from black widow spider venom. Toxicon 2007, 49:531-549.

25. Graudins A, Little MJ, Pineda SS, Hains PG, King GF, Broady KW, Nicholson GM: Cloning and activity of a novel a-latrotoxin from red-back spider venom Biochem Pharmacol 2012, 83:170-183.

26. Garb JE, Hayashi CY: Molecular evolution of alpha-Latrotoxin, the exceptionally potent vertebrate neurotoxin in black widow spider venom. Mol Biol Evol 2013, 30:999-1014.

27. Ushkaryov YA, Volynski KE, Ashton AC: The multiple actions of black widow spider toxins and their selective use in neurosecretion studies. Toxicon 2004, 43:527-542.

28. Pescatori M, Bradbury A, Bouet F, Gargano N, Mastrogiacomo A, Grasso A: The cloning of a cDNA encoding a protein (latrodectin) which co-purifies with the alpha-latrotoxin from the black widow spider Latrodectus tredecimguttatus (Theridiidae). Eur J Biochem 1995, 230:322-328.

29. Volkova TM, Pluzhnikov KA, Woll PG, Grishin EV: Low molecular weight components from black widow spider venom. Toxicon 1995, 33:483-489.

30. Marioni JC, Mason CE, Mane SM, Stephens M, Gilad Y: RNA-seq: An assessment of technical reproducibility and comparison with gene expression arrays. Genome Res 2008, 18:1509-1517.

31. Yuan C, Jin Q, Tang X, Hu W, Cao R, Yang S, Xiong J, Xie C, Xie J, Liang S: Proteomic and peptidomic characterization of the venom from the Chinese bird spider, Ornithoctonus huwena Wang. J Proteome Res 2007, 6:2792-2801.

32. Tang $X$, Zhang $Y, H u$ W, Xu D, Tao H, Yang X, Li Y, Jiang L, Liang S: Molecular diversification of peptide toxins from the tarantula Haplopelma hainanum (Ornithoctonus hainana) venom based on transcriptomic, peptidomic, and genomic analyses. J Proteome Res 2010, 9:2550-2564.

33. Maretić Z: Latrodectism: variations in clinical manifestations provoked by Latrodectus species of spiders. Toxicon 1983, 21:457-466.

34. Isbister GK, Gray MR: Latrodectism: a prospective cohort study of bites by formally identified redback spiders. Med J Aust 2003, 179:88-91.

35. Silva J-P, Suckling J, Ushkaryov Y: Penelope's web: using a-latrotoxin to untangle the mysteries of exocytosis. J Neurochem 2009, 111:275-290.

36. Huang X: CAP3: a DNA sequence assembly program. Genome Res 1999, 9:868-877.
37. Clarke TH, Garb JE, Hayashi CY, Haney RA, Lancaster AK, Corbett S, Ayoub NA: Multi-tissue transcriptomics of the black widow spider reveals expansions, co-options, and functional processes of the silk gland gene toolkit. BMC Genomics 2014, 15:365.

38. Grishin EV: Black widow spider toxins: the present and the future. Toxicon 1998, 36:1693-1701.

39. Li J, Mahajan A, Tsai M-D: Ankyrin repeat: a unique motif mediating protein-protein interactions. Biochemistry (Mosc) 2006, 45:15168-15178.

40. Von Reumont BM, Blanke A, Richter S, Alvarez F, Bleidorn C, Jenner RA: The first venomous crustacean revealed by transcriptomics and functional morphology: remipede venom glands express a unique toxin cocktail dominated by enzymes and a neurotoxin. Mol Biol Evol 2014, 31:48-58.

41. Jungo F, Bairoch A: Tox-Prot, the toxin protein annotation program of the Swiss-Prot protein knowledgebase. Toxicon 2005, 45:293-301.

42. Coddington JA: Phylogeny and classification of spiders. In Spiders N Am Identif Man. American Arachnological Society; 2005:18-24.

43. Vassilevski AA, Fedorova IM, Maleeva EE, Korolkova YV, Efimova SS, Samsonova OV, Schagina LV, Feofanov AV, Magazanik LG, Grishin EV: Novel class of spider toxin: active principle from the yellow sac spider Cheiracanthium punctorium venom is a unique two-domain polypeptide. J Biol Chem 2010, 285:32293-32302.

44. Kuhn-Nentwig L, Fedorova IM, Lüscher BP, Kopp LS, Trachsel C, Schaller J, Vu XL, Seebeck T, Streitberger K, Nentwig W, Sigel E, Magazanik LG: A venom-derived neurotoxin, CsTx-1, from the spider Cupiennius salei exhibits cytolytic activities. J Biol Chem 2012, 287:25640-25649.

45. Sollod BL, Wilson D, Zhaxybayeva O, Gogarten JP, Drinkwater R, King GF: Were arachnids the first to use combinatorial peptide libraries? Peptides 2005, 26:131-139.

46. Südhof TC: alpha-Latrotoxin and its receptors: neurexins and CIRL/ latrophilins. Annu Rev Neurosci 2001, 24:933-962.

47. Rowen L, Young J, Birditt B, Kaur A, Madan A, Philipps DL, Qin S, Minx P, Wilson RK, Hood L, Graveley BR: Analysis of the human neurexin genes: alternative splicing and the generation of protein diversity. Genomics 2002, 79:587-597.

48. Reissner C, Runkel F, Missler M: Neurexins. Genome Biol 2013, 14:213.

49. Finkelstein A, Rubin LL, Tzeng MC: Black widow spider venom: effect of purified toxin on lipid bilayer membranes. Science 1976, 193:1009-1011.

50. Mironov SL, Sokolov YV, Chanturiya AN, Lishko VK: Channels produced by spider venoms in bilayer lipid membrane: mechanisms of ion transport and toxic action. Biochim Biophys Acta 1986, 862:185-198.

51. Wagh DA, Rasse TM, Asan E, Hofbauer A, Schwenkert I, Dürrbeck H, Buchner S, Dabauvalle M-C, Schmidt M, Qin G: Bruchpilot, a protein with homology to ELKS/CAST, is required for structural integrity and function of synaptic active zones in Drosophila. Neuron 2006, 49:833-844.

52. Zipursky SL, Wojtowicz WM, Hattori D: Got diversity? Wiring the fly brain with Dscam. Trends Biochem Sci 2006, 31:581-588.

53. Hortsch M, Nagaraj K, Godenschwege TA: The interaction between L1-type proteins and ankyrins - a master switch for L1-type CAM function. Cell Mol Biol Lett 2008, 14:57-69.

54. Laurén J, Airaksinen MS, Saarma M, Timmusk T: A novel gene family encoding leucine-rich repeat transmembrane proteins differentially expressed in the nervous system. Genomics 2003, 81:411-421.

55. Grueber WB, Yang C-H, Ye B, Jan Y-N: The development of neuronal morphology in insects. Curr Biol 2005, 15:R730-R738.

56. Duda TF, Palumbi SR: Molecular genetics of ecological diversification: duplication and rapid evolution of toxin genes of the venomous gastropod Conus. Proc Natl Acad Sci 1999, 96:6820-6823.

57. Chen J, Zhao L, Jiang L, Meng E, Zhang Y, Xiong X, Liang S: Transcriptome analysis revealed novel possible venom components and cellular processes of the tarantula Chilobrachys jingzhao venom gland. Toxicon 2008, 52:794-806.

58. Sedgwick SG, Smerdon SJ: The ankyrin repeat: a diversity of interactions on a common structural framework. Trends Biochem Sci 1999, 24:311-316

59. Zhang D, de Souza RF, Anantharaman V, lyer LM, Aravind L: Polymorphic toxin systems: comprehensive characterization of trafficking modes, processing, mechanisms of action, immunity and ecology using comparative genomics. Biol Direct 2012, 7:18.

60. Perret BA: Proteolytic activity of tarantula venoms due to contamination with saliva. Toxicon Off J Int Soc Toxinology 1977, 15:505-510.

61. Matsui T, Fujimura Y, Titani K: Snake venom proteases affecting hemostasis and thrombosis. Biochim Biophys Acta 2000, 1477:146-156. 
62. Smith D, Russell F: Structure of the venom gland of the black widow spider Latrodectus mactans. A preliminary light and electron microscopic study. In Anim Toxins. Edited by Russell F, Saunders P. Oxford: Pergamon; 1966:1-15.

63. Vetter RS, Isbister GK: Medical aspects of spider bites. Annu Rev Entomol 2008, 53:409-429.

64. Grabherr MG, Haas BJ, Yassour M, Levin JZ, Thompson DA, Amit I, Adiconis X, Fan L, Raychowdhury R, Zeng Q, Chen Z, Mauceli E, Hacohen N, Gnirke A, Rhind N, di Palma F, Birren BW, Nusbaum C, Lindblad-Toh K, Friedman N, Regev A: Full-length transcriptome assembly from RNA-Seq data without a reference genome. Nat Biotechnol 2011, 29:644-652.

65. Haas BJ, Papanicolaou A, Yassour M, Grabherr M, Blood PD, Bowden J, Couger MB, Eccles D, Li B, Lieber M, MacManes MD, Ott M, Orvis J, Pochet N, Strozzi F, Weeks N, Westerman R, William T, Dewey CN, Henschel R, LeDuc RD, Friedman N, Regev A: De novo transcript sequence reconstruction from RNA-seq using the Trinity platform for reference generation and analysis. Nat Protoc 2013, 8:1494-1512.

66. Altschul SF, Gish W, Miller W, Myers EW, Lipman DJ: Basic local alignment search tool. J Mol Biol 1990, 215:403-410.

67. Li B, Dewey CN: RSEM: accurate transcript quantification from RNA-Seq data with or without a reference genome. BMC Bioinformatics 2011, 12:323.

68. Langmead B, Trapnell C, Pop M, Salzberg SL: Ultrafast and memoryefficient alignment of short DNA sequences to the human genome. Genome Biol 2009, 10:R25.

69. Finn RD, Clements J, Eddy SR: HMMER web server: interactive sequence similarity searching. Nucleic Acids Res 2011, 39(suppl):W29-W37.

70. Young MD, Wakefield MJ, Smyth GK, Oshlack A: Gene ontology analysis for RNA-seq: accounting for selection bias. Genome Biol 2010, 11:R14.

71. Quevillon E, Silventoinen V, Pillai S, Harte N, Mulder N, Apweiler R, Lopez R: InterProScan: protein domains identifier. Nucleic Acids Res 2005, 33(Web Server):W116-W120.

72. Naamati G, Askenazi M, Linial M: ClanTox: a classifier of short animal toxins. Nucleic Acids Res 2009, 37(Web Server):W363-W368.

73. Kaplan N, Morpurgo N, Linial M: Novel families of toxin-like peptides in insects and mammals: a computational approach. J Mol Biol 2007, 369:553-566.

74. Gracy J, Le-Nguyen D, Gelly J-C, Kaas Q, Heitz A, Chiche L: KNOTTIN: the knottin or inhibitor cystine knot scaffold in 2007. Nucleic Acids Res 2007, 36(Database):D314-D319

75. Emanuelsson O, Brunak S, von Heijne G, Nielsen H: Locating proteins in the cell using TargetP, SignalP and related tools. Nat Protoc 2007, 2:953-971.

76. Wolters DA, Washburn MP, Yates JR: An automated multidimensional protein identification technology for shotgun proteomics. Anal Chem 2001, 73:5683-5690.

77. Eng JK, McCormack AL, Yates JR: An approach to correlate tandem mass spectral data of peptides with amino acid sequences in a protein database. J Am Soc Mass Spectrom 1994, 5:976-989.

78. Papadopoulos JS, Agarwala R: COBALT: constraint-based alignment tool for multiple protein sequences. Bioinformatics 2007, 23:1073-1079.

79. Capella-Gutierrez S, Silla-Martinez JM, Gabaldon T: trimAl: a tool for automated alignment trimming in large-scale phylogenetic analyses. Bioinformatics 2009, 25:1972-1973.

80. Ronquist F, Teslenko M, van der Mark P, Ayres DL, Darling A, Hohna S, Larget B, Liu L, Suchard MA, Huelsenbeck JP: MrBayes 3.2: efficient Bayesian phylogenetic inference and model choice across a large model space. Syst Biol 2012, 61:539-542.

81. Stamatakis A: RAxML version 8: a tool for phylogenetic analysis and post-analysis of large phylogenies. Bioinformatics 2014, 30:1312-1313.

82. McCowan C, Garb JE: Recruitment and diversification of an ecdysozoan family of neuropeptide hormones for black widow spider venom expression. Gene 2014, 536:366-375.

doi:10.1186/1471-2164-15-366

Cite this article as: Haney et al: Dramatic expansion of the black widow toxin arsenal uncovered by multi-tissue transcriptomics and venom proteomics. BMC Genomics 2014 15:366.

\section{Submit your next manuscript to BioMed Central and take full advantage of:}

- Convenient online submission

- Thorough peer review

- No space constraints or color figure charges

- Immediate publication on acceptance

- Inclusion in PubMed, CAS, Scopus and Google Scholar

- Research which is freely available for redistribution

Submit your manuscript at www.biomedcentral.com/submit
() Biomed Central 